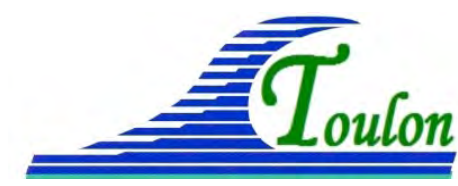

XIV $V^{\text {èmes }}$ Journées Nationales Génie Côtier - Génie Civil Toulon, 29 juin au $1^{\text {er }}$ juillet 2016

DOI:10.5150/jngcgc.2016.070 C Editions Paralia CFL disponible en ligne - http://www.paralia.fr - available online

\title{
Comparaison du coût de différentes mesures de protection de la vie humaine face au risque de submersion marine
}

\author{
Axel CREACH ${ }^{1,3}$, Emilio BASTIDAS-ARTEAGA ${ }^{2}$, \\ Sophie PARDO ${ }^{3}$, Denis MERCIER ${ }^{4}$
}

1. Université de Nantes, IGARUN, Laboratoire LETG-Nantes Géolittomer, UMR 6554 CNRS, Campus du Tertre, BP 81227, 44312 Nantes, France.

Axel.Creach@univ-nantes.fr

2. Université de Nantes, Institut de Recherche en Génie Civil et Mécanique (GeM), UMR 6183 CNRS, 2 rue de la Houssinière, BP 92208, 44322 Nantes, France. Emilio.Bastidas@univ-nantes.fr

3. Université de Nantes, Institut d'Economie et de Management de Nantes Campus du Tertre, BP 52231, 44312 Nantes, France.

Sophie.Pardo@univ-nantes.fr

4. Université Paris-Sorbonne (Paris IV), UFR de Géographie et d'Aménagement, Laboratoire ENeC, UMR 8185 CNRS, 191, rue Saint Jacques, 75005 Paris, France. Denis.Mercier@paris-sorbonne.fr

\section{Résumé :}

En février 2010, la tempête Xynthia a été à l'origine du décès de 41 personnes, suite à la submersion de zones littorales basses. Les retours d'expérience ont notamment mis en lumière l'urbanisation récente de zones potentiellement inondables et l'inadaptation architecturale des constructions résidentielles. Face à l'existence de ces zones, l'Etat a décidé de déconstruire les habitations présentant une exposition trop importante pour leurs occupants. Cette politique des "zones noires" a été coûteuse et controversée. Cette communication propose une revue de mesures alternatives, et de leurs coûts, dans la perspective de réaliser une analyse Coût-Efficacité, a posteriori, de ces mesures confrontées aux "zones noires" établies suite à la tempête Xynthia. Ces mesures sont : (i) la protection, (ii) l'adaptation architecturale, (iii) l'alerte et la prévention et (iv) la relocalisation.

Mots-clés : Risques littoraux, Submersion marine, Vulnérabilité, Adaptation, Protection, Digues, Xynthia.

\section{Introduction}

Dans la nuit du 27 au 28 février 2010, la tempête Xynthia a frappé les côtes atlantiques françaises, de l'estuaire de la Loire à l'estuaire de la Gironde. La conjonction de vents forts et d'une marée haute de coefficient 102 est à l'origine de niveaux marins exceptionnels sur les côtes de Vendée et de Charente-Maritime (BERTIN et al., 2012). 50000 hectares de zones littorales basses ont été submergés par la mer (VERGER, 


\section{Thème 7 - Risques côtiers}

2011) dont une partie était occupée par l'urbanisation dont la vulnérabilité était connue (RAISON, 2008). Cette submersion de zones urbanisées a conduit au décès par noyade de 41 personnes (VINET et al., 2011), dont 93\% sont intervenus à l'intérieur de constructions résidentielles.

Les retours d'expérience de l'événement Xynthia ont mis en avant l'importance de la localisation et de la configuration du bâti résidentiel dans l'explication du bilan humain de la tempête (VINET et al., 2011) en particulier que (i) 100\% des décès sont intervenus dans des constructions où le niveau d'eau a été supérieur à 1 mètre ; (ii) $90 \%$ des décès ont été localisés dans des constructions situées à moins de 400 mètres des digues; et (iii) 78\% des décès ont été enregistrés dans des constructions de plain-pied.

Ainsi, le bâti peut conduire à une vulnérabilité "extrême" des occupants en cas d'événement de submersion marine, pouvant aller jusqu'au décès (CREACH et al., 2015).

Suite à la tempête Xynthia, l'Etat a décidé d'identifier, de racheter et de détruire les constructions présentant un risque potentiellement mortel pour les occupants à travers la politique des zones noires ou zones de solidarité. Cette mesure a été critiquée pour sa mise en œuvre précipitée, les critères utilisés pour les délimiter (MERCIER \& CHADENAS, 2012), et sa cherté (COUR DES COMPTES, 2012). Ces zones noires s’apparentent à une mesure de "relocalisation", stratégie onéreuse à court terme mais radicale dans l'objectif de protéger la vie humaine face au risque de submersion marine. La question de la mise en œuvre de mesures alternatives se pose néanmoins : plutôt que la destruction systématique des constructions, n'aurait-il pas été possible de rehausser les digues afin de protéger les constructions les plus exposées ? L’ajout systématique d'un étage aux constructions de plain-pied ne permettrait-il pas aux populations de se réfugier?

Dans l'objectif de mener une analyse économique de mesures alternatives de réduction de la vulnérabilité sur sept communes du littoral atlantique français (Noirmoutier-enl'Île, Barbâtre, La Guérinière, L'Epine, La Faute-sur-Mer, L'Aiguillon-sur-Mer et Charron), ce travail s'attache à chiffrer individuellement le coût de différentes actions permettant d'agir sur cette vulnérabilité. Ces actions sont évidemment complémentaires et ont vocation à être couplées afin d'élaborer des stratégies globales de réduction du niveau de risque.

Dans un premier temps, les principales mesures de réduction de la vulnérabilité sont identifiées. Une présentation plus détaillée des mesures retenues est ensuite proposée, avec présentation des coûts nécessaires pour leur mise en œuvre.

\section{Les mesures d'adaptation au changement climatique}

Afin de réduire la vulnérabilité du bâti résidentiel et de protéger les populations face au risque de submersion marine, plusieurs stratégies peuvent être adoptées. Classiquement, dans le cadre des recherches menées sur l'adaptation à l'élévation du niveau marin liée 


\section{XIV ${ }^{\text {èmes }}$ Journées Nationales Génie Côtier - Génie Civil \\ Toulon, 29 juin au $1^{\text {er }}$ juillet 2016}

au changement climatique, trois grands types de stratégies sont proposés (PASKOFF, 2001 ; KLEIN et al., 2001 ; NICHOLLS, 2011 ; GIEC, 2014). Celles-ci sont décrites dans le tableau 1.

Pour ce travail, une déclinaison de ces trois stratégies sera étudiée : la protection, la relocalisation et deux mesures d'accommodation: l'adaptation architecturale des constructions pour permettre la mise en sécurité des populations, et la prévention et l'évacuation préventive, permettant de réduire le nombre d'individus présents dans la zone à risque en cas d'événement de submersion marine imminent.

Tableau 1. Principales stratégies d'adaptation à l'élévation du niveau marin liée au changement climatique (source : NICHOLLS, 2011).

Stratégie d'adaptation
Protection : réalisation d'une structure de protection, dont
l'objectif sera de réduire l'occurrence de l'aléa sur un secteur
donné en contenant le niveau marin, l'impact des vagues et des
franchissements.
Déplacement : retrait des enjeux les plus exposés à travers la
relocalisation (MEDDE, 2012) en-dehors des zones d'aléa.
C'est notamment ce qui a été fait à travers les "zones noires"
post-Xynthia.
Accommodation : action permettant de minimiser l'impact de "dur "
l'aléa tout en maintenant les enjeux à leur place initiale.

\section{Mesure de protection}

\subsection{Objectif}

La mesure consiste à maintenir artificiellement la position du trait de côte, soit de manière fixe avec la réalisation d'une digue ou d'un mur de protection, soit de manière plus ou moins souple en ayant recours à des pratiques telles le réensablement (PASKOFF, 2000 ; NICHOLLS, 2011). Ainsi, elle permet de réduire la probabilité de l'aléa de submersion marine en contenant les niveaux marins au droit d'une zone basse (KLEIN et al., 2001). Néanmoins, ces ouvrages peuvent connaître des défaillances (rupture ou surverse en cas de sous-dimensionnement, de mauvais entretien ou de dégradation dans le temps). Certains auteurs pointent également le coût exponentiel de leur adaptation à l'élévation du niveau marin liée au changement climatique (JONKMAN et al., 2013) ainsi que le sentiment de sécurité erroné qu'ils procurent aux personnes vivant en arrière de ces ouvrages (VINET, 2010). 


\section{Thème 7 - Risques côtiers}

C’est la mesure pourtant la plus couramment adoptée dans les pays développés (KLEIN et al., 2001).

\subsection{Mise en œuvre}

Dans ce travail, seuls les ouvrages déjà existants seront traités en envisageant leur confortement et leur rehaussement afin d'être dimensionnés de façon à tenir compte de l'élévation du niveau marin liée au changement climatique. C'est une mesure qui relève généralement de l’action publique, particulièrement des collectivités.

\subsection{Coût}

DOLLET et SYLLA (2015) ont chiffré le coût associé au confortement d'une portion de digue sur l'ile de Noirmoutier et à son rehaussement. Ce rehaussement de $120 \mathrm{~cm}$ consistait à porter la crête de digue à une altitude supérieure de $20 \mathrm{~cm}$ aux plus hautes eaux connues en intégrant une marge de $60 \mathrm{~cm}$ pour tenir compte de l'élévation du niveau marin liée au changement climatique. La totalité de ces travaux a été estimée à $500 €$ par ml, auxquels il convient d'ajouter une provision annuelle pour entretien équivalent à $2 \%$ de l’investissement, soit $10 €$ par ml par an.

Bien que cette fourchette soit similaire avec d'autres études (GOELDNER GIANELLA \& BERTRAND, 2013), il convient de nuancer ces coûts qui peuvent varier en fonction de l'état initial de l'ouvrage, de la rehausse nécessaire ou de la proximité des matériaux à mettre en œuvre pour ces travaux.

\section{Mesure de relocalisation}

\section{$4.1 \underline{\text { Objectif }}$}

Dans certains cas, le niveau d'exposition est tel que les mesures de protection s'avèrent trop onéreuses dans leur mise en œuvre, et que les mesures d'accommodation ne peuvent garantir une réelle baisse de la vulnérabilité. Le déplacement des enjeux en dehors de la zone d'aléa s'impose donc.

C'est une mesure qui connaît des précédents, notamment pour des enjeux exposés au recul du trait de côte où le déplacement peut devenir inévitable (KLEIN et al., 2001). Pour ce qui est du risque de submersion marine, cette approche est encore nouvelle, les zones noires post-Xynthia faisant figure de pionnières en France. Cette politique avait conduit au rachat et à la destruction de 1628 habitations jugées trop dangereuses pour leurs occupants, pour un total de 315,7 millions d'euros.

Dans un contexte d'élévation du niveau marin liée au changement climatique, cette stratégie devrait devenir inévitable à l'avenir comme le souligne la stratégie nationale de gestion du trait de côte (MEDDE, 2012). 


\section{XIV èmes Journées Nationales Génie Côtier - Génie Civil \\ Toulon, 29 juin au $1^{\text {er }}$ juillet 2016}

\subsection{Mise en œuvre}

Du point de vue de la mise en œuvre, cette mesure nécessite le rachat et la déconstruction des habitations les plus exposées. Cela fait de cette stratégie une option chère à court terme mais qui rend nuls les dommages futurs.

Elle relève de l'action publique dans le cadre des rachats amiables ou expropriations menées dans le cadre du fond Barnier.

\subsection{Coût}

Compte tenu de la pression foncière sur les communes littorales, il est proposé d'estimer un coût de rachat des constructions spécifique à chaque commune (méthode testée sur les sept communes étudiées). Pour ce faire, un coût moyen au $\mathrm{m}^{2}$ par commune est calculé à partir de différents sites internet à défaut de pouvoir accéder aux coûts des transactions (http://www.meilleursagents.com/prix-immobilier, http://priximmobilier.drimki.fr et Efficity.com). Sur l'ensemble des communes étudiées, ce coût est en moyenne de $2366 €$ par $\mathrm{m}^{2}$, avec un écart-type de $555 €$. Ce coût peut ensuite être multiplié par la surface des constructions devant être relocalisées.

Pour ce qui est du coût de déconstruction, les données des coûts post-Xynthia n’ont pas été accessibles. Celui-ci a donc été estimé à $20000 €$ par construction, ce qui correspond à la fourchette moyenne proposée par le site :

http://www.renovationettravaux.fr/cout-demolition-maison-prix-devis.

Ces coûts sont à nuancer du fait en l'absence d'autres sources de données permettant de confirmer les fourchettes proposées. De plus, ils pourraient être complétés en intégrant le coût de renaturation des sites ainsi déconstruits.

\section{Mesure d'accommodation : l'adaptation architecturale}

\section{$5.1 \underline{\text { Objectif }}$}

Cette mesure est une forme "d'accommodation" puisqu'elle consiste à maintenir les enjeux dans leur position initiale et à les adapter afin de réduire la vulnérabilité des personnes au risque de submersion marine. Concrètement, plusieurs solutions sont envisageables :

- les mesures consistant à rehausser le niveau du premier plancher : mise sur pilotis, mise sur butte, constructions flottantes, etc. ;

- les mesures permettant d'offrir une zone de refuge : aménagement de combles ou ajout d'un étage refuge.

Ces différentes solutions n’offrent pas la même temporalité, les premières sont plus adaptées aux constructions futures tandis que les secondes relèvent de mesures applicables aux constructions existantes. 


\section{Thème 7 - Risques côtiers}

\subsection{Mise en œuvre}

Dans le présent travail, seule l'adaptation architecturale des constructions existantes sera présentée, et plus particulièrement à la construction d'un étage refuge tel que proposé par le Référentiel de travaux de prévention du risque d'inondation dans l'habitat existant (MEDDE \& METL, 2012 et figure 1).

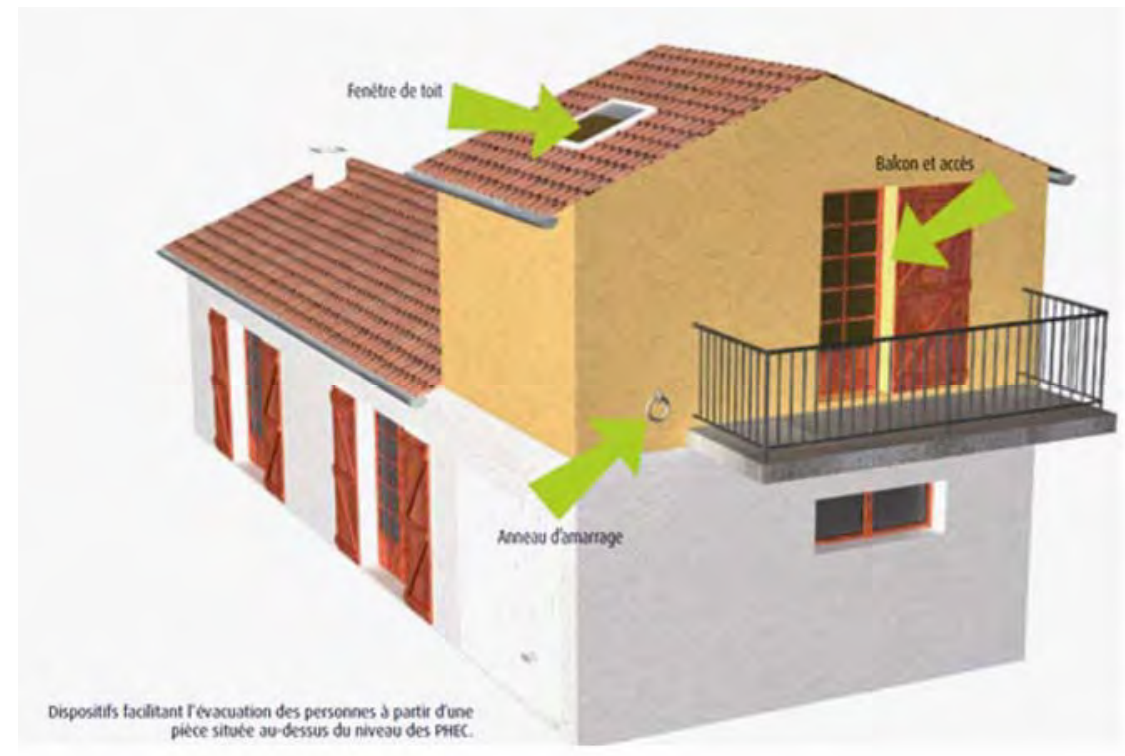

Figure 1. Exemple de la création d'un étage refuge (source : MEDDE \& METL, 2012).

Signalons toutefois que cette mesure ne garantit pas une mise en sécurité totalement efficace des individus en cas de submersion marine puisque, dans certains cas, ils peuvent être pris au piège au rez-de-chaussée malgré la présence d'un étage. Lors de Xynthia, $17 \%$ des décès sont intervenus dans des constructions possédant un étage (VINET et al., 2011).

Cette action relève d'initiatives individuelles mais peut être supportée par des incitations financières.

\subsection{Coût}

Le coût de l'aménagement d'un étage refuge type, tel que proposé par le Référentiel de travaux de prévention du risque d'inondation dans l'habitat existant (MEDDE et METL, 2012 et figure 1), a été estimé à $19 \mathrm{k} €$ par construction (FESTUOT \& GUILLAUME, 2015). 


\section{XIV ${ }^{\text {èmes }}$ Journées Nationales Génie Côtier - Génie Civil \\ Toulon, 29 juin au $1^{\text {er }}$ juillet 2016}

\section{Mesure d'accommodation : prévention, alerte et évacuation préventive}

\subsection{Objectif}

Plutôt que d'agir sur l'adaptation architecturale des constructions, on peut aussi vouloir limiter la population présente en zone à risque en cas d'événement de submersion marine.

Cette mesure vise à limiter temporairement les enjeux humains exposés en favorisant l'évacuation préventive en cas d'alerte d'un événement de submersion marine. C'est une mesure qui peut être particulièrement efficace (PARKER et al., 2009) et comme l'ont souligné KOLEN et al. (2013), une telle évacuation aurait permis de limiter le bilan humain de la tempête Xynthia. Néanmoins, l'efficacité de l'évacuation va dépendre du nombre de personnes qui vont effectivement évacuer, qui peut être extrêmement variable (PRIEST et al., 2007), mais également du temps disponible entre le début de l'alerte et la survenue de l'événement et de l'organisation matérielle de l'évacuation.

La première étape pour envisager un taux d'évacuation acceptable en cas d'alerte est de s'assurer que la population soit consciente du risque et soit préparée à y faire face, c'està-dire à adopter de bons comportements. Cela passe par des actions de prévention. La difficulté d'une telle mesure est qu'il faut maintenir la conscience du risque, c’est donc un processus permanent.

\section{2 $\underline{\text { Mise en œuvre }}$}

Dans cette mesure, deux volets sont envisagés :

- la mise en place de mesures de prévention, permettant l'information et la formation de la population, à travers la réalisation de documents réglementaires d'information et de prévention (Plan de Prévention des Risques, Document d'Information Communal sur les Risques Majeurs, Plan Communal de Sauvegarde) et la réalisation d'exercices d'évacuation de façon régulière. Ces différentes actions permettraient d'augmenter le taux d'évacuation en cas d'alerte.

- L’évacuation totale d'une zone étant illusoire, il peut être pertinent de rendre obligatoire l'équipement des ménages en "Kit d'attente des secours" (Plus d'informations et liste des objets composant ce "kit" sur http://www.developpementdurable.gouv.fr/Kit-d-attente-des-secours-pendant.html) tel que recommandé par le ministère de l'écologie, ainsi que d'installer des plateformes refuges permettant de se réfugier en dernier recours. Différentes solutions de telles plateformes existent (CREACH, 2015).

Ces différentes actions relèvent de l'action publique. 


\section{Thème 7 - Risques côtiers}

\subsection{Coût}

Les actions de prévention en tant que telles sont envisagées à l'échelle communale. Les coûts de réalisation des différents documents et des exercices d'évacuation sont tirés du Plan d'Action et de Prévention des Inondations (PAPI) de la communauté d'agglomération de La Rochelle (CDA LA ROCHELLE, 2013). Ils se montent à $357000 €$ par commune. Une mise à jour tous les cinq ans est envisagée afin de maintenir la conscience du risque.

L'installation de plateformes refuges rendue obligatoire par certains PAPI dans les campings et leur installation est de l'ordre de $80000 €$ selon le prestataire privé qui en propose différents modèles (Entreprise TDEM). Ce coût est variable selon la taille et l'équipement de la structure. Enfin, l'équipement de foyers en "Kit d'attente des secours" est estimé à un coût unitaire de $250 €$.

Tableau 2. Récapitulatif des différentes mesures

\begin{tabular}{|c|c|c|c|c|}
\hline Mesure & Description & Coût d'investissement & $\begin{array}{l}\text { Coût d'entretien } \\
\text { (provision annuelle) }\end{array}$ & Source \\
\hline Protection & $\begin{array}{l}\text { Rehaussement et confortements } \\
\text { des ouvrages de protection } \\
\text { existants (pas de nouveaux } \\
\text { ouvrages) }\end{array}$ & Digue $=500 €$ par ml & $\begin{array}{l}\text { Digue }=2 \% \text { du coût } \\
\text { d'investissement ( } 10 € \\
\text { par ml/an) }\end{array}$ & $\begin{array}{l}\text { DOLLET \& SYLLA } \\
\text { (2015) }\end{array}$ \\
\hline $\begin{array}{l}\text { Adaptation } \\
\text { architecturale }\end{array}$ & $\begin{array}{l}\text { Construction d'une pièce } \\
\text { refuge pour les constructions } \\
\text { de plain-pied. Mise en place } \\
\text { d'une fenêtre de toit pour } \\
\text { permettre une évacuation } \\
\text { verticale. }\end{array}$ & $19265 €$ par construction & Considéré nul & $\begin{array}{l}\text { FESTUOT \& } \\
\text { GUILLAUME } \\
\text { (2015) }\end{array}$ \\
\hline \multirow{2}{*}{$\begin{array}{l}\text { Prévention, } \\
\text { alerte et } \\
\text { évacuation }\end{array}$} & $\begin{array}{l}\text { Faciliter l'évacuation } \\
\text { préventive de la population } \\
\text { exposée en cas d'alerte en } \\
\text { l'informant (documents }\end{array}$ & $\begin{array}{l}\text { Documents d'information et de } \\
\text { réglementation } L=307000 € \\
\text { Exercices d'évacuation }= \\
50000 €\end{array}$ & $\begin{array}{l}\text { Prévention = mise à jour } \\
\text { des documents et } \\
\text { réalisation d'exercice } \\
\text { d'évacuation tous les } 5 \\
\text { ans }=71450 €\end{array}$ & $\begin{array}{l}\text { CDA LA } \\
\text { ROCHELLE (2013) }\end{array}$ \\
\hline & $\begin{array}{l}\text { formant (exercice } \\
\text { d'évacuation), et offrir la } \\
\text { possibilité de refuge en dernier } \\
\text { recours (plateforme refuge). }\end{array}$ & $\begin{array}{l}\text { Plateforme refuge (unité) = } \\
80000 € \\
\text { Kit d'attente des secours } \\
\text { pendant } 3 \text { jours (coût par } \\
\text { foyer) }=250 €\end{array}$ & $\begin{array}{l}\text { Plateforme refuge }=1 \% \\
\text { du coût d'investissement } \\
\text { (800 € par an) }\end{array}$ & Entreprise TDEM \\
\hline \multirow[b]{2}{*}{ Relocalisation } & Suppression des constructions & $\begin{array}{l}\text { Rachat }=\text { coût au } m^{2} \text { estimé } \\
\text { par commune }\end{array}$ & \multirow[b]{2}{*}{ Nul dans le temps } & $\begin{array}{l}\text { Rachat = sélection } \\
\text { de sites internet }\end{array}$ \\
\hline & $\begin{array}{l}\text { rachat et la destruction de ces } \\
\text { habitations }\end{array}$ & $\begin{array}{l}\text { Déconstruction }=20000 € \text { par } \\
\text { construction }\end{array}$ & & $\begin{array}{l}\text { Déconstruction }= \\
\text { http://www.renovati } \\
\text { onettravaux.fr/ }\end{array}$ \\
\hline
\end{tabular}




\section{XIV vèmes Journées Nationales Génie Côtier - Génie Civil \\ Toulon, 29 juin au $1^{\text {er }}$ juillet 2016}

\section{Conclusions}

L'objectif de cette communication était d'identifier le coût de différentes mesures de réduction de la vulnérabilité du bâti résidentiel et de leurs occupants face au risque de submersion marine. Le tableau 2 propose un récapitulatif de ces mesures, de leur coût d'investissement mais également de leur coût d'entretien.

Malgré les incertitudes relatives à ces chiffres, il est possible d'estimer le coût global de la mise en œuvre de différentes stratégies de réduction de la vulnérabilité à l'échelle d'une commune, associant une ou plusieurs des mesures présentées ici. C'est une étape essentielle afin de pouvoir comparer l'intérêt de ces scénarios au travers d'une analyse de type coût-avantage telle qu'elle a été menée pour sept communes de la façade atlantique française (CREACH, 2015) et ainsi aboutir à un outil d'aide à la décision pour la mise en place de stratégies de réduction de la vulnérabilité de l'habitat résidentiel et de ses occupants.

\section{Références bibliographiques}

BERTIN X., BRUNEAU N., BREILH J-F., FORTUNATO A., KARPYTCHEV M. (2012). Importance of wave age and resonance in storm surges: The case Xynthia, Bay of Biscay. Ocean Modelling, Vol. 42, pp 16-30. http://dx.doi.org/10.1016/j.ocemod.2011.11.001 CDA LA ROCHELLE (2013). Programme d'Action de Prévention des Inondations "Agglomération Rochelaise". Communauté d'agglomération de La Rochelle.

COUR DES COMPTES (2012). Les enseignements des inondations de 2010 sur le littoral atlantique (Xynthia) et dans le Var. Rapport public thématique.

CREACH A. (2015). Cartographie et analyse économique de la vulnérabilité du littoral atlantique français face au risque de submersion marine (2 volumes). Thèse Université de Nantes. Disponible en ligne sur URL : https://tel.archives-ouvertes.fr/tel-01275600.

CREACH A., PARDO S., GUILLOTREAU P., MERCIER D. (2015). The use of a micro-scale index to identify potential death risk areas due to coastal flood surges: lessons from Storm Xynthia on the French Atlantic coast. Natural Hazards, Vol. 77(3), pp 1679-1710. http://dx.doi.org/10.1007/s11069-015-1669-y

DOLLET V., SYLLA O. (2015). Techniques de renforcement et coûts d'adaptation des digues face à des nouveaux risques en lien avec le changement climatique - Application au cas de l'Ile de Noirmoutier. Mémoire d'initiation à la recherche, Master Génie Civil, Université de Nantes.

FESTUOT R., GUILLAUME L. (2015). Etude technico économique sur la réduction de la vulnérabilité des constructions de plain-pied soumises à des risques de submersions. Mémoire d'initiation à la recherche, Master Génie Civil, Université de Nantes.

GIEC (2014). Climate Change 2014: Impacts, Adaptation and Vulnerability. GIEC/IPCC.

GOELDNER GIANELLA L., BERTRAND F. (2013). BARCASUB : La submersion marine et ses impacts environnementaux et sociaux dans le Bassin d'Arcachon 
(France): est-il possible, acceptable et avantageux de gérer ce risque par la dépoldérisation ? Rapport final, Programme LITEAU III

JONKMAN S., HILLEN M., NICHOLLS R., KANNING W., VAN LEDDEN, M. (2013). Costs of Adapting Coastal Defences to Sea-Level Rise- New Estimates and Their Implications. Journal of Coastal Research, Vol. 29(5), pp 1212-1226. http://dx.doi.org/10.2112/JCOASTRES-D-12-00230.1

KLEIN R., NICHOLLS R., RAGOONADEN S., CAPOBIANCO M., ASTON J., BUCKLEY E. (2001). Technological Options for Adaptation to Climate Change in Coastal Zones. Journal of Coastal Research, Vol. 17(3), pp 531-543.

KOLEN B., SLOMP R., JONKMAN S. (2013). The impacts of storm Xynthia February 27-28, 2010 in France: lessons for flood risk management. Journal of Flood Risk Management. Vol. 6(3), pp 261-278. http://dx.doi.org/10.1111/jfr3.12011

MEDDE -Ministère de l'Écologie, du Développement durable et de l’Énergie- (2012). Stratégie nationale de gestion intégrée du trait de côte - Vers la relocalisation des activités et des biens. Rapport.

MEDDE, METL (2012). Référentiel de travaux de prévention du risque d'inondation dans l'habitat existant. Rapport.

MERCIER D., CHADENAS C. (2012). La tempête Xynthia et la cartographie des "zones noires" sur le littoral français : analyse critique à partir de l'exemple de La Faute-sur-Mer (Vendée). Norois, Vol. 222(1), pp 45-60. http://dx.doi.org/10.4000/norois.3895

NICHOLLS R. (2011). Planning for the Impacts of Sea Level Rise. Oceanography, Vol. 24(2), pp 144-157. http://dx.doi.org/10.5670/oceanog.2011.34

PARKER D., PRIEST S., TAPSELL S. (2009). Understanding and enhancing the public's behavioural response to flood warning information. Meteorological Applications; Vol. 16(1), pp 103-114. http://dx.doi.org/10.1002/met.119

PASKOFF R. (2001). L'élévation du niveau de la mer et les espaces côtiers. Institut Océanographique, Paris, 190 p.

PRIEST S., WILSON T., TAPSELL S., PENNING-ROWSELL E., VIAVATTENE C., FERNANDEZ-BILBAO A. (2007). T10-07-10: Building a model to estimate Risk to Life for European flood events. Rapport final, Middlesex University.

RAISON S. (2008). Le classement des digues littorales au titre de la sécurité civile : un exemple de mise en auvre en Vendée. Journées Nationales Génie Côtier - Génie Civil, Sophia Antipolis, pp 283-292. http://dx.doi.org/10.5150/ingcgc.2008.027-R

VERGER F. (2011). Digues et polders littoraux : réflexions après la tempête Xynthia, Physio-Géo, Vol. 5, pp 95-105. http://dx.doi.org/10.4000/physio-geo.1740

VINET F. (2010). Le risque inondation. Diagnostic et gestion. Lavoisier, Paris, 318 p. VINET F., BOISSIER L., DEFOSSEZ S. (2011). La mortalité comme expression de la vulnérabilité humaine face aux catastrophes naturelles : deux inondations récentes en France (Xynthia, Var, 2010). VertigO, Vol. 11(2), 28 p. http://dx.doi.org/10.4000/vertigo.11074 